\title{
Surgical anatomy of the superior hypophyseal artery and its relevance for endoscopic endonasal surgery
}

\author{
Huy Q. Truong, MD, Edinson Najera, MD, Robert Zanabria-Ortiz, MD, Emrah Celtikci, MD, \\ Xicai Sun, MD, PhD, Hamid Borghei-Razavi, MD, PhD, Paul A. Gardner, MD, and \\ Juan C. Fernandez-Miranda, MD
}

Department of Neurological Surgery, University of Pittsburgh School of Medicine, Pittsburgh, Pennsylvania

\begin{abstract}
OBJECTIVE The endoscopic endonasal approach has become a routine corridor to the suprasellar region. The superior hypophyseal arteries (SHAs) are intimately related to lesions in the suprasellar space, such as craniopharyngiomas and meningiomas. Here the authors investigate the surgical anatomy and variations of the SHA from the endoscopic endonasal perspective.
\end{abstract}

METHODS Thirty anatomical specimens with vascular injection were used for endoscopic endonasal dissection. The number of SHAs and their origin, course, branching, anastomoses, and areas of supply were collected and analyzed.

RESULTS A total of 110 SHAs arising from 60 internal carotid arteries (ICAs), or 1.83 SHAs per ICA (range 0-3), were found. The most proximal SHA always ran in the preinfundibular space and provided the major blood supply to the infundibulum, optic chiasm, and proximal optic nerve; it was defined as the primary SHA (pSHA). The more distal SHA(s), present in $78.3 \%$ of sides, ran in the retroinfundibular space and supplied the stalk and may also supply the tuber cinereum and optic tracts. In the two sides (3.3\%) in which no SHA was present, the territory was covered by a pair of infundibular arteries originating from the posterior communicating artery. Two-thirds of the pSHAs originated proximal to the distal dural ring; half of these arose from the carotid cave portion of the ICA, whereas the other half originated proximal to the cave. Four branching patterns of the pSHA were recognized, with the most common pattern (41.7\%) consisting of three or more branches with a tree-like pattern. Descending branches were absent in $25 \%$ of cases. Preinfundibular anastomoses between pSHAs were found in all specimens. Anastomoses between the PSHA and the secondary SHA (SSHA) or the infundibular arteries were found in $75 \%$ cases.

CONCLUSIONS The first SHA almost always supplies the infundibulum, optic chiasm, and proximal optic nerve and represents the pSHA. Compromising this artery can cause a visual deficit. Unilateral injury to the pSHA is less likely to cause an endocrine deficit given the artery's abundant anastomoses. A detailed understanding of the surgical anatomy of the SHA and its many variations may help surgeons when approaching challenging lesions in the suprasellar region. https://thejns.org/doi/abs/10.3171/2018.2.JNS172959

KEYWORDS superior hypophyseal artery; suprasellar; carotid cave; infundibulum; pituitary surgery; anatomy

$\mathrm{O}$ VER the last decade, the endoscopic endonasal approach has become a routine surgical corridor for suprasellar lesions..$^{24,27}$ The superior hypophyseal arteries (SHAs), which provide critical blood supply for the infundibulum, adenohypophysis, optic chiasm, and nerves, ${ }^{2,4,6,9,11,23,25,29}$ are intimately related to lesions in the suprasellar space, such as craniopharyngiomas and meningiomas, and there is often a need to dissect, mobilize, or even sacrifice the SHA or its branches to obtain adequate tumor access and removal. ${ }^{19}$

Previous anatomical studies on SHAs have been based on either a microscopic transcranial view or sectioned blocks of the sellar region, , ,4,6,6,11,19-21,23,25,28,29 with unavoidable retraction and alteration of the neurovascular structures and their spatial relationships during the investigative procedure. Authors have also noted the difficulties

ABBREVIATIONS DDR = distal dural ring; ICA = internal carotid artery; ICG = indocyanine green; PCoA = posterior communicating artery; $\mathrm{pSHA}=$ primary SHA; SHA = superior hypophyseal artery; SSHA = secondary SHA.

SUBMITTED November 23, 2017. ACCEPTED February 2, 2018.

INCLUDE WHEN CITING Published online July 13, 2018; DOI: 10.3171/2018.2.JNS172959. 
in accessing the infrachiasmatic space for dissecting and studying the deep-seated and delicate SHAs. ${ }^{6,9}$ In this study, we investigated the surgical anatomy and variations of the SHA from the endoscopic endonasal perspective, and we describe the surgical nuances for safe and effective mobilization and preservation of the SHA when performing endoscopic endonasal surgery in the suprasellar space.

\section{Methods}

Thirty human head specimens with colored-silicone vascular injection were used for dissection. The endoscopic endonasal transsellar-transtuberculum approach was performed to widely expose the suprasellar area, as previously described. ${ }^{5}$ The number of SHAs and their origin, course, branching, anastomoses, and areas of supply were collected and analyzed. Distal dural rings (DDRs) and carotid collars were dissected when necessary to inspect the carotid caves ${ }^{13}$ and the origin of the SHA. Pituitary gland transposition, as described in detail in another paper, ${ }^{8}$ was performed when necessary to access the lateral recess of the interpeduncular cistern and confirm the location and trajectory of the posterior communicating artery (PCoA). We categorized every artery that arose from the internal carotid artery (ICA) and passed to the infundibulum as an SHA. When branches to the infundibulum arose from the PCoAs, they were defined as "infundibular arteries," and when they arose from the ophthalmic artery, they were named "prechiasmal arteries," as described previously.

\section{Results}

We found a total of 110 SHAs among 196 perforators arising from 60 ICAs, with an average of 1.83 SHAs per ICA (range 0-3). In addition, we identified 12 infundibular arteries arising from 10 PCoAs (17\%) and 1 prechiasmal artery $(1.7 \%)$ during the dissection.

\section{Arrangement of SHAs}

In 42 (70\%) of 60 sides, there were two SHAs arising from the ICA: the first or most proximal one ran in the preinfundibular space to supply the optic nerve, optic chiasm, and infundibulum, whereas the second or distal one ran in the retroinfundibular space or lateral to the stalk (parainfundibular) to reinforce the supply to the infundibulum and occasionally supply the tuber cinereum, optic tracts, or even the mammillary bodies (Fig. 1). We defined them as primary and secondary SHAs (pSHA and sSHA), respectively. In 5 sides (8.3\%), there was a third SHA that contributed to the vasculature of the infundibulum. In 11 sides (18.3\%), there was only 1 SHA, which was either a large artery sending branches along both the pre- and retroinfundibular space or a common trunk that bifurcated close to its origin from the ICA in a V-shaped manner into a pSHA and an sSHA accordingly (Fig. 1D). Among these hemispheres with a single SHA, there were 4 with infundibular arteries that took the course and irrigating area of an sSHA and 1 with a prechiasmal artery that served the area of a pSHA. Here we introduce the concept of the infundibular and prechiasmal arteries as complementary blood supply to the infundibulum and optic nerve, respec- tively, when the "proper" SHA, which by definition originates directly from the ICA, is not present. In 2 sides, there were no strictly defined SHAs, and the territory was covered by a pair of infundibular arteries from the PCoA (Fig. 1D) that irrigated the infundibulum and optic chiasm. In 5 sides, the infundibular arteries supplied the infundibulum to complement the pSHA and sSHA.

\section{Origin of pSHA}

We found that only one-third of the pSHAs originated from the ophthalmic segment of the ICA distal to the DDR (Fig. 1C), whereas the other two-thirds originated from the clinoidal segment of the ICA proximal to the DDR. Among the latter pSHAs, approximately half originated from the carotid cave, a small dural recess between the wall of the ICA and the posteromedial aspect of the DDR (Fig. 1B), ${ }^{13,16,18}$ whereas the other half originated proximal to the cave, running within the tissue of the carotid collar and reaching the subarachnoid space via the carotid cave (Fig. 1A).

\section{Branching Pattern of the PSHA}

The pSHA provided a wide variation of branches and areas of blood supply. We classified the branches into three main groups: infundibular, optic, and descending (Fig. 2). Infundibular branches were always present and run medially and posteriorly to reach the upper infundibulum directly and mingle with their counterparts to form a preinfundibular anastomosis and send out small perforators to supply the stalk, median eminence, and adjacent chiasm. Optic branches run superiorly to supply the ventral and anterior optic chiasm or curve back to follow and feed the ventral cisternal segment of the optic nerve (also known as recurrent ${ }^{16}$ or ascending branches ${ }^{9}$ ) or run posteriorly to supply the ventral aspect of the anterior portion of the optic tract; they were present in all hemispheres except one. Descending branches run downward in the arachnoid layer alongside the stalk, either well attached or at a distance from the stalk, toward the upper surface of the gland and sellar diaphragm. The descending branch was previously referred to as the "loral artery" by McConnell"23 and "artery of the trabecular" by Xuereb et al. ${ }^{29}$ These branches were absent in $25 \%$ of cases, and when present, they supplied the sellar diaphragm in $50 \%$ of cases (meningeal branches), the anterior lobe of the pituitary gland and distal pituitary stalk in 43\%, and both in 7\% (Fig. 3A-C). There were $4(6.7 \%)$ of 60 cases in which the descending branches originated from the sSHAs instead of the pSHA.

The pSHA was found to send off additional meningeal branches to supply the prechiasmatic sulcus and planum sphenoidale dura in $15 \%$ of cases. These branches could arise as a direct branch from the pSHA or as a smaller branch from optic or infundibular branches that pierced through the arachnoid membranes to reach the dura (Fig. 3D).

We broadly defined four branching patterns of the pSHA: 1) candelabra-like type (30\%), the main artery splits off to form three groups of branches (infundibular, optic, and descending) at approximately the same point (Figs. 2B and $4 \mathrm{~A}) ; 2$ ) tree-like or early optic-branching type (41.7\%), 

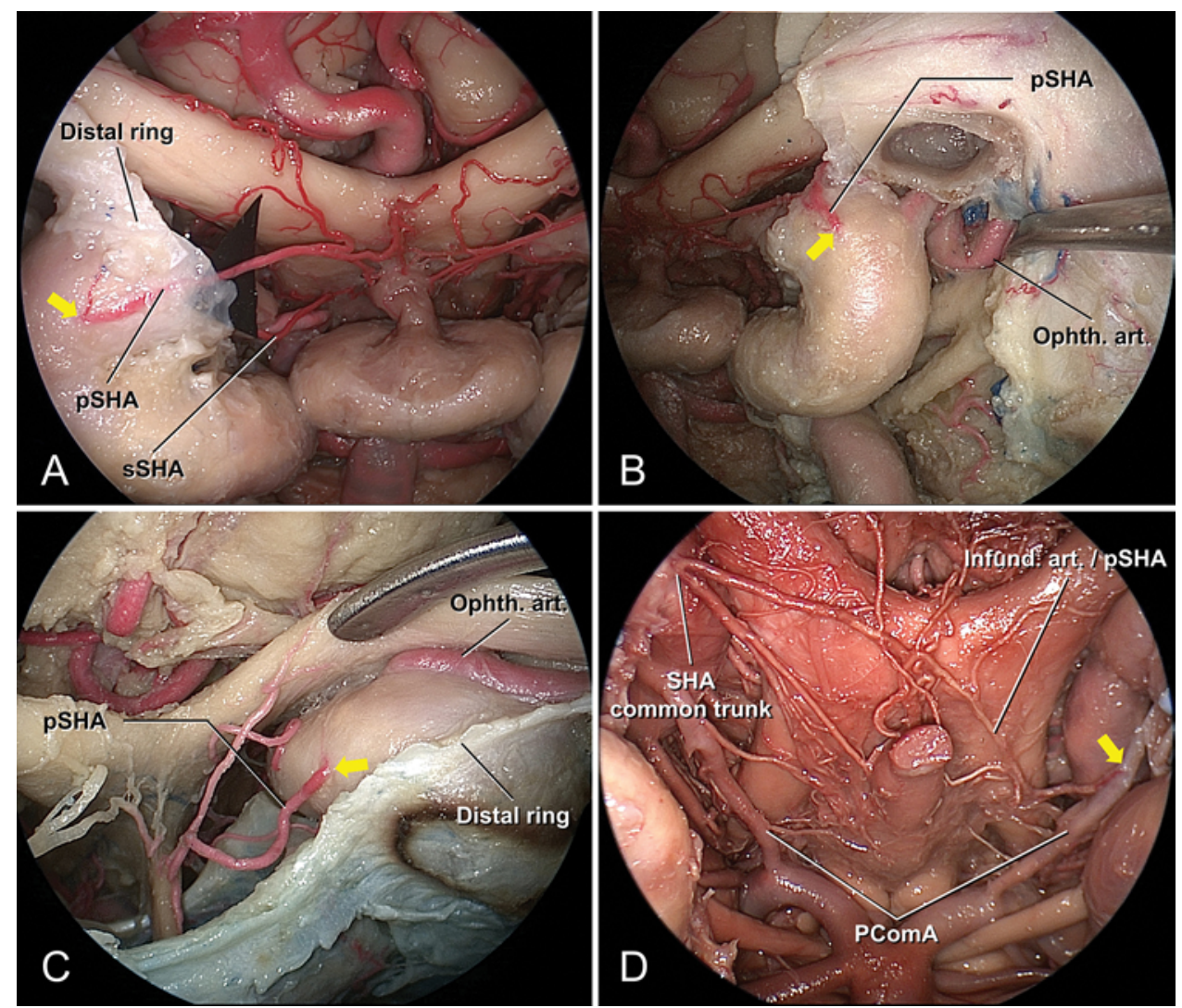

FIG. 1. Origin and pattern of the superior hypophyseal artery (SHA). A: The primary SHA (pSHA) originates from the clinoidal segment of the ICA (yellow arrow), proximal to the carotid cave, demonstrated by the black plastic, and runs in the preinfundibular space. The secondary SHA (sSHA) can be seen running toward the posterior part of the infundibulum. B: The pSHA with a carotid cave origin (yellow arrow) and the cavernous sinus ophthalmic artery. C: The pSHA (yellow arrow) branches off from the ophthalmic segment of the ICA. D: Infundibular artery, considered as a variant of the PSHA in this study (see text), arises from the PCoA on the left side (yellow arrow) and runs to the preinfundibular area whose supplying territory is similar to a pSHA. On the right, the first two SHAs take off as a common trunk at the ophthalmic segment of the ICA. art. = artery; Infund. = infundibular; Ophth. = ophthalmic; PComA = posterior communicating artery. Copyright Juan C. Fernandez-Miranda. Published with permission. Figure is available in color online only.

the branches take off one by one from the main trunk, and most of the time, the optic branches are first and the descending branches tend to take off from the infundibular branches (Figs. 2C and 4A); 3) no-descending type (25\%), where the descending branch is not present and the pSHA is not anchored inferiorly and runs closer to the optic nerve and chiasm (Figs. 2D and 4B); and 4) no-optic type (1.7\%), where there is no optic branch from the pSHA, a rare variant that was found in only one case over 60 pSHAs (Figs. $2 \mathrm{E}$ and $4 \mathrm{~B})$.

\section{Dominance and Anastomosis}

A macroscopically visible and significant side dominance of the pSHA was found in $8(27 \%)$ of 30 cases (Fig. $5 \mathrm{~A}$ ). Anastomoses with the contralateral SHA were found in all cases; in the vast majority $(90 \%)$, the anastomosis was formed between infundibular branches from both sides. In 2 cases the anastomosis was formed between the pSHA and the contralateral sSHA, and in 1 case it was developed between infundibular and optic branches. In 2 cases with no "proper" SHA, the anastomoses were formed between the pSHA on one side and the substitute on the other side, which was the infundibular artery in those cases (Fig. 1D). In all cases, the anastomoses were formed anterior to the infundibulum (Figs. 1B and 5A). We suggest the term "preinfundibular anastomosis" to more accurately describe this particular and constant vascular structure and to differentiate it from the well-known circuminfundibular anastomosis, ${ }^{6,9}$ which additionally includes less frequent anastomoses between SHAs of the same side, with perforators from the PCoA (infundibular arteries), and retroinfundibular anastomoses between contralateral sSHAs (Fig. 5B and C). Additionally, 2 cases of large interhypophyseal anastomoses were found between descending branches from the sSHAs and the ipsilateral inferior hypophyseal artery (IHA) through the diaphragm (Fig. 5D). Both descending arteries were branches of sSHAs. This variation may fit the definition of middle hypophyseal artery per some authors. ${ }^{3,20}$

\section{Surgical Nuances}

Case 1: Anterior Displacement: Craniopharyngioma

A 30-year-old female presented with a 6-month history of amenorrhea and intermittent headache. A CT scan 

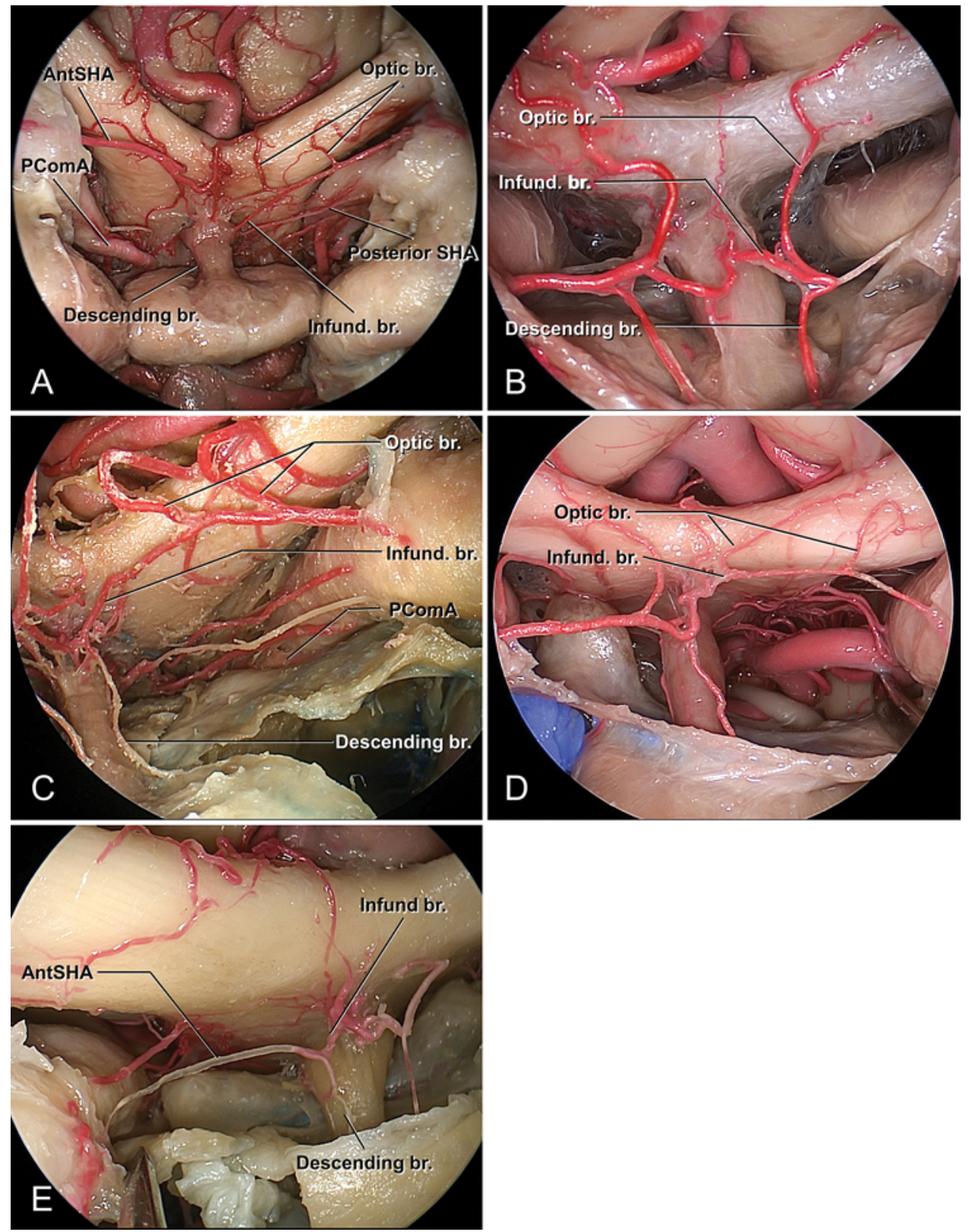

FIG. 2. A: Types of branching of the pSHA: infundibular, optic, and descending. B: Pattern of branching: candelabra-like type, where the three major branches are taking off from the main trunk at approximately the same point. C: Pattern of branching: treelike or early optic-branching type, four optic branches can be seen alternatingly branching off the pSHA before two infundibular branches. A descending branch comes off a larger infundibular branch. D: Pattern of branching: no-descending type, on the left side, only two optic and one infundibular branch can be seen; there is no descending from the left pSHA. E: Pattern of branching: no-optic type, right pSHA shows a descending branch and an infundibular branch with no branch to the optic nerve. Of note, abundant supply can be seen originating from the anterior communicating complex. br. = branch; Infund. = infundibular. Copyright Juan C. Fernandez-Miranda. Published with permission. Figure is available in color online only.

showed a calcified and partially cystic lesion in the suprasellar space. Magnetic resonance imaging revealed a heterogeneously enhanced suprasellar lesion with compression of the optic apparatus and cystic extension into the third ventricle. Mild hyperprolactinemia was found, but her pituitary function was otherwise normal. Surgical treatment was recommended. An endoscopic endonasal transsellar and transtuberculum approach was performed. The tumor was found to be a highly calcified lesion at- tached to the pituitary stalk and extending to the retrochiasmatic space. A pSHA was found on the left, running on the suprasellar arachnoid layer anteriorly and superiorly to the tumor, consistent with a tree-like or early opticbranching pattern, with one large descending branch well attached to the anterior surface of the tumor (Fig. 6A). Dissection was difficult as the pSHA blocked access to the retrochiasmatic part of the tumor. The descending branch was then selectively coagulated and sacrificed, 

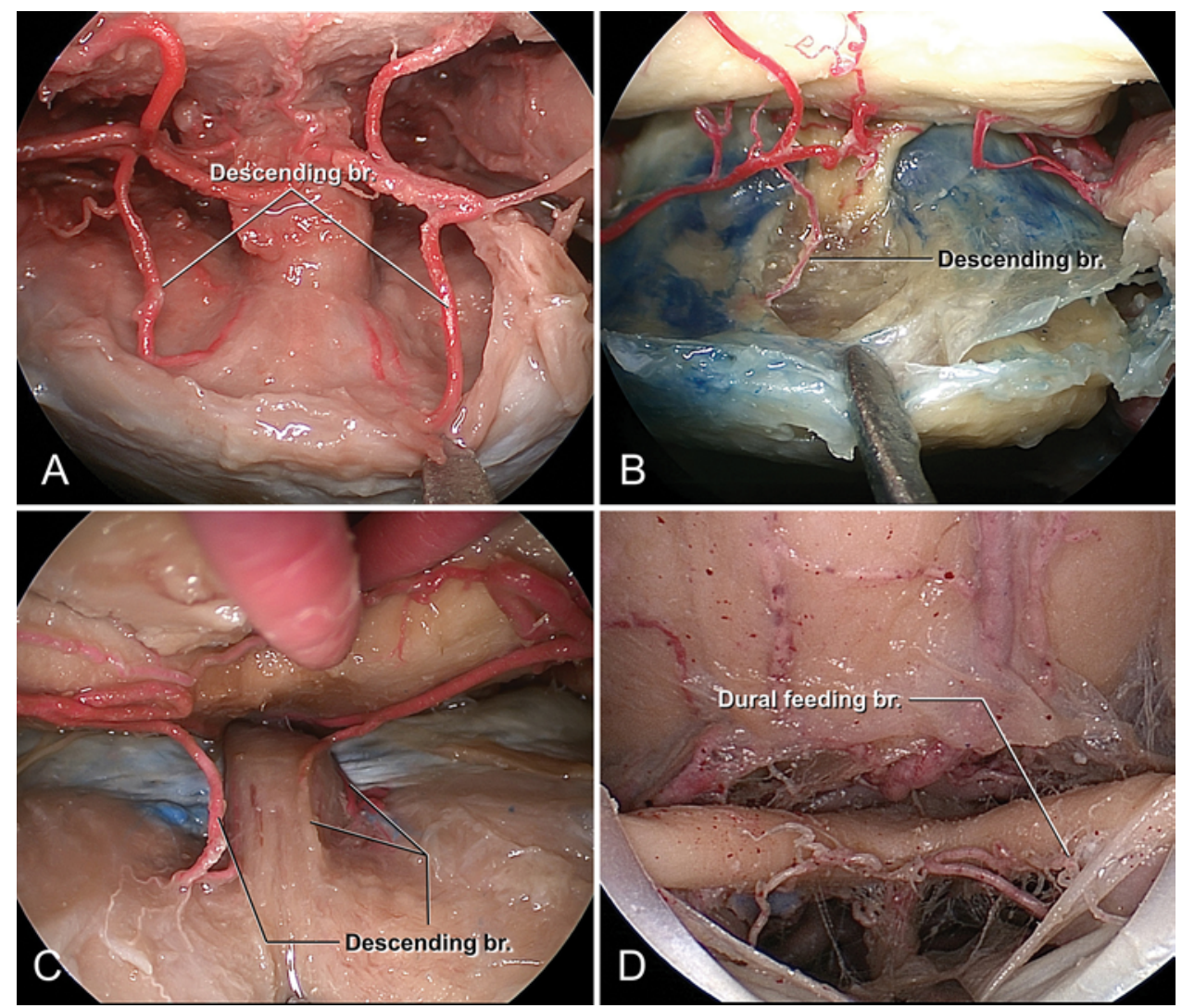

FIG. 3. Descending branch and dural feeding. A: Two descending branches reach the anterior lobe of the pituitary gland and course back to the junction of the gland and the stalk to supply both. B: Descending branch feeding the diaphragm dura. C: The descending branch from the right side feeds both the dura and the gland. D: A small vessel branching off the right optic branch, piercing the arachnoid membrane to reach and feed the prechiasmatic sulcus dura. br. = branch. Copyright Juan C. FernandezMiranda. Published with permission. Figure is available in color online only.

detaching the pSHA and allowing its dissection superiorly off the tumor surface. The calcified portion was then dissected from the stalk and removed (Fig. 6B). Complete tumor resection, including the cyst wall extending within the third ventricle, was accomplished. The patient developed diabetes insipidus and adrenal insufficiency after surgery. Pathological findings confirmed the diagnosis of adamantinomatous craniopharyngioma. Amenorrhea and diabetes insipidus resolved at 3 months' follow-up, but the patient remained on hydrocortisone replacement (Video 1).

VIDEO 1. Clip of clinical cases 1 and 2. Copyright Juan C.

Fernandez-Miranda. Published with permission. Click here to view.

\section{Case 2: Posterior Displacement: Tuberculum Sella Meningioma}

A 43-year-old male was incidentally diagnosed with a sellar tumor during a workup following a minor head trauma. Further physical examination revealed a mild temporal hemianopia in the left eye. Magnetic resonance imaging showed a homogeneously enhancing, extraaxial lesion in the prechiasmatic cistern, compressing the left optic nerve. An endoscopic endonasal transsellar-transtuberculum approach was performed. After the dura was opened, a dissection plane between the right tumor edge and the arachnoid was established. After initial debulking, the pituitary stalk, together with SHAs from both sides, was identified (Fig. 6C). Tumor removal was advanced toward the left side with care taken to preserve all posteriorly displaced SHA branches, which appeared to be the early optic-branching pattern (Fig. 6D). The patient experienced no new neurological deficits postoperatively and had a reversal of his visual defect (Video 1).

Case 3: Adherence/Encasement: Pituitary Adenoma With Suprasellar Extension and Subarachnoidal Invasion

A 51-year-old male had been suffering from progressive visual loss for 9 months. Physical examination revealed visual acuity was 20/400 in the left eye and 20/30 in the right eye with a homonymous hemianopia. Imaging showed a homogeneously enhanced sellar lesion with suprasellar and retrochiasmatic extension with significant compression of the optic chiasm. Laboratory workup revealed mild hypothyroidism. An endoscopic endonasal transsellar-transplanum approach was performed. The suprasellar portion of the tumor pushed the right descending SHA branch anteriorly and the optic and infundibular branches posteriorly but with significant adherence to the tumor capsule. The descending branch was identified going to the diaphragm and was sacrificed; this facilitated tumor dissection of the other branches, which were meticulously preserved (Fig. 6E and F). The left SHA 


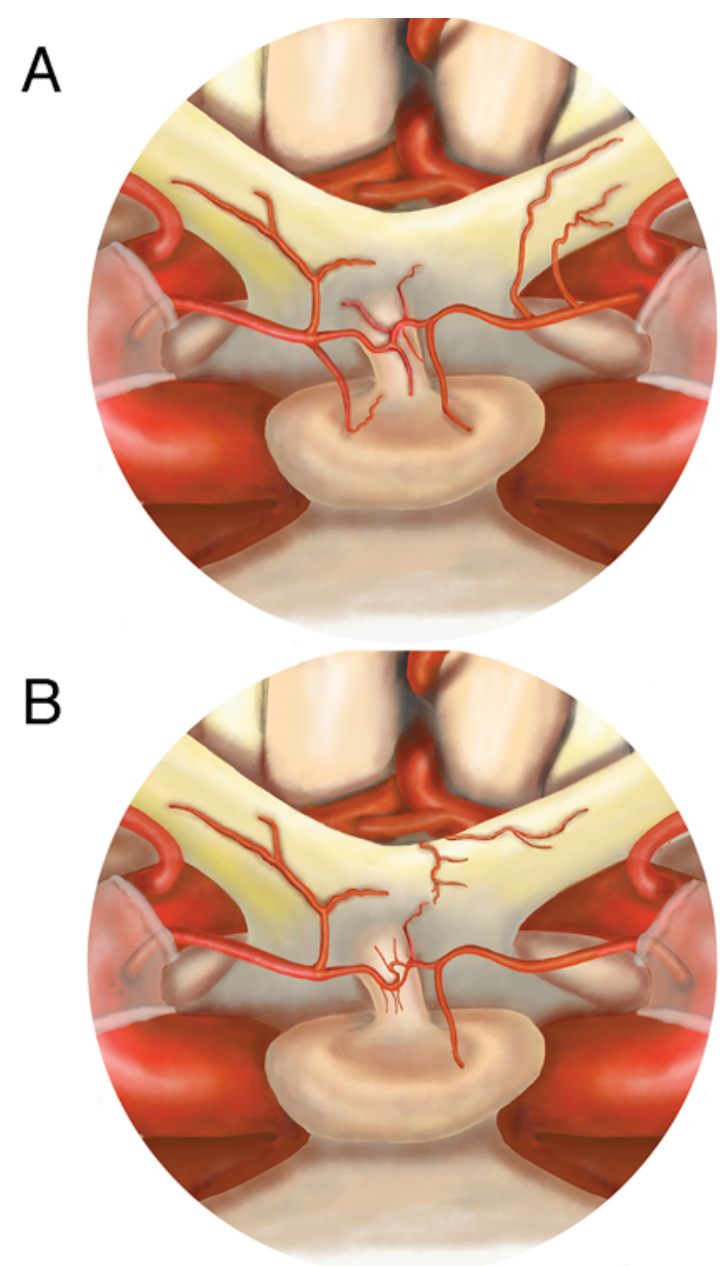

FIG. 4. Artistic illustration of branching patterns of SHA. A: Type A, the candelabra-like type, is illustrated on the right side, and type $B$, the tree-like or early optic-branching type, is on the left. B: Type C, the nodescending type, is on the right side, and type $\mathrm{D}$, the no-optic type, is on the left. Copyright Juan C. Fernandez-Miranda. Published with permission. Figure is available in color online only.

branches were also adherent and partially encased but were preserved by leaving a thin rim of tumor capsule that fused with the infundibulum. The patient did well postoperatively with no new deficits and visual improvement. Histopathological examination confirmed the diagnosis of pituitary adenoma (Video 2).

VIDEO 2. Clip of clinical case 3. Copyright Juan C. FernandezMiranda. Published with permission. Click here to view.

\section{Discussion}

Multiple authors have described the SHAs as branches of the ICA distal to the DDR and proximal to the origin of the PCoA. Kobayashi et al., however, suggested that the SHA may originate from a pouch invaginating between the ICA and adjacent DDR, which they named the "carotid cave." 18 The potential origin of the SHA from the carotid cave was later confirmed by several authors. ${ }^{13,16,17}$ Our findings confirm that the first or most proximal SHA, which is almost universally the pSHA, is more likely to arise from the ICA proximal to the DDR (clinoidal segment), with only one-third originating distal to it (ophthalmic segment). Care should be taken when working on the clinoidal ICA, especially with bipolar coagulation, to avoid accidentally damaging the pSHA at its origin.

The pSHA, with its forefront position and critical irrigating area, has significant clinical relevance during the surgical treatment of suprasellar lesions. Based on our surgical experience, cystic lesions that originate from the pituitary stalk, such as craniopharyngiomas and suprasellar Rathke's cleft cysts, tend to push the suprasellar arachnoid layer and pSHA forward in the way of surgical access to the tumor. This arrangement can hinder total tumor removal or make it more challenging and may be the reason that hypopituitarism can result even with anatomical stalk preservation. Similarly, meningiomas of the tuberculum sella typically have a tendency to push the pSHA posteriorly together with the suprasellar arachnoid and chiasm. In these cases, it is critical to find the suprasellar arachnoid plane to preserve the pSHA branches. Meningiomas from the diaphragma sellae and large adenomas with subarachnoidal invasion have a more complex and unpredictable relation to the pSHA, which can be displaced upward, forward, or backward, depending on the tumor growth pattern, with actual vascular encasement in many instances. All these surgical scenarios place the pSHA at risk; therefore, it is of significant clinical interest to fully understand the potential branches and branching pattern of the SHAs to better identify them and predict the consequences of compromising the pSHA or its branches, either uni- or bilaterally.

In this study, we found that the SHA can provide meningeal branches to the prechiasmatic sulcus and planum dura and that these can be a source of early bleeding right after the dura is opened during a transtuberculum approach because the vessels are anchored to the arachnoid and easily torn. These branches can be enlarged in cases of meningioma and should be selectively coagulated.

Based on our findings, the infundibulum can receive blood supply from 4 or more arteries in $80 \%$ of cases. The preinfundibular anastomoses were present in all cases with reinforcement from sSHAs or infundibular arteries from the PCoA in many cases (75\%). This feature is in agreement with previous authors ${ }^{6,10,19,21-23,29}$ and makes the infundibulum and pituitary function less vulnerable to unilateral compromise of the pSHA. The endocrinological outcome of SHA occlusion is rarely found in the literature. There is, however, an interesting report on the anatomical outcome of pituitary stalk transection, a procedure that was quite popular in the 1960s to treat advanced breast cancer, prostate cancer, and diabetic retinopathy. ${ }^{1}$ The authors examined the necropsies of 21 pituitary glands of patients who survived the stalk transection surgery at various times. In most of them there was some band of surviving epithelial tissue, and close to the posterior lobe. Transection of the pituitary stalk is definitely more devastating than occlusion of 2 or even 3 SHAs. Moreover, the minimal literature reporting on pituitary dysfunction after SHA occlusion implies the high tolerance of the pituitary stalk and gland to the partial loss of blood sources.

The median portion of the optic chiasm receives blood supply from both preinfundibular anastomosis branches 

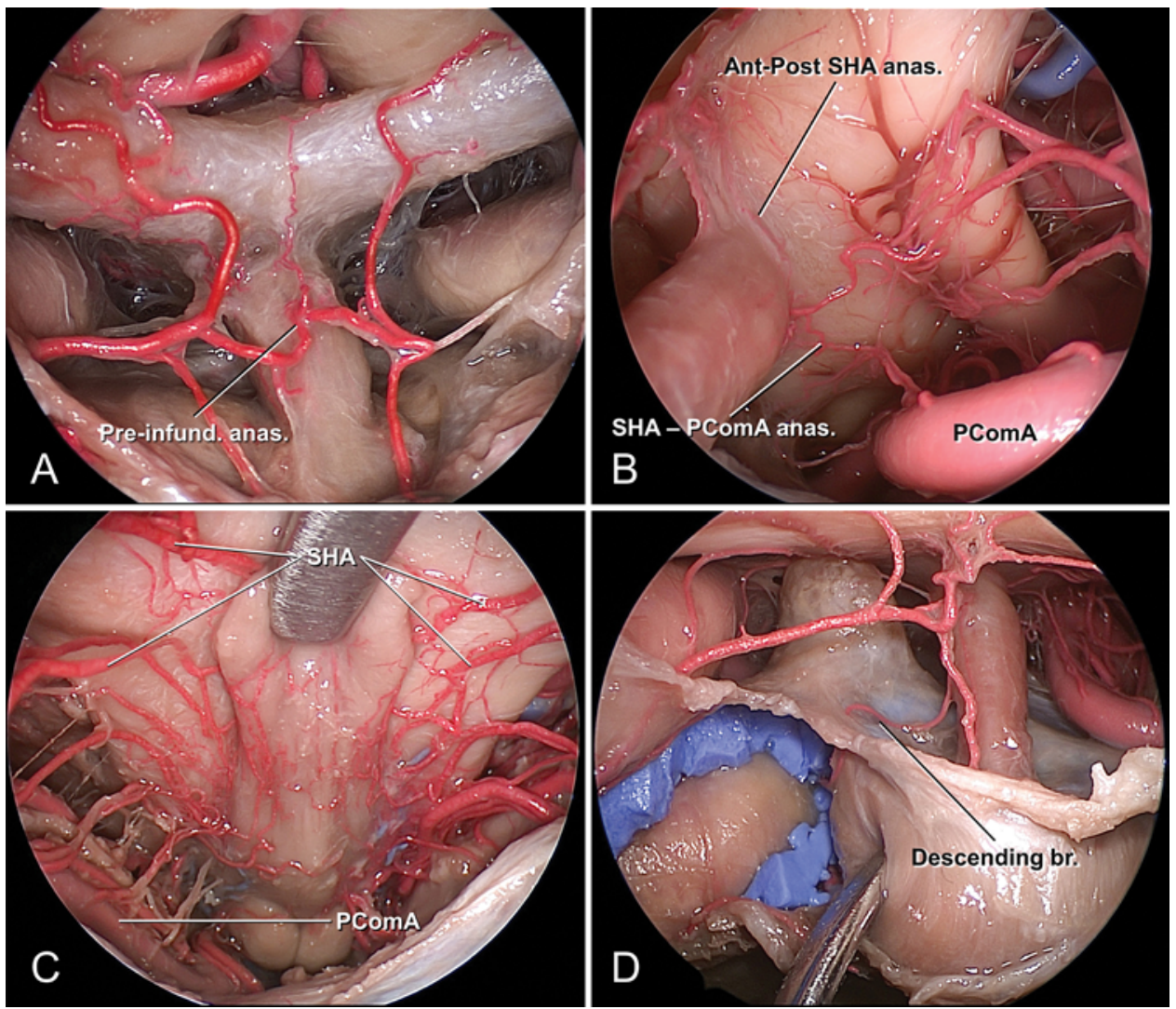

FIG. 5. Anastomoses of SHA. A: Preinfundibular anastomosis, a prominent side-to-side anastomosis of the two pSHAs. The right pSHA appears to be significantly dominant to the left one. B: The anastomosis between the pSHA and sSHA of the same side and that between posterior SHA and perforators from the PCoA help to reinforce the preinfundibular anastomosis and comprise the circuminfundibular anastomosis. C: Retroinfundibular anastomosis between sSHA and perforator from the PCoA. D: A descending branch, in this case from the SSHA, pierces through the diaphragm to connect to a branch from the inferior hypophyseal artery (IHA) to form an extrahypophyseal SHA-IHA anastomosis. anas. = anastomosis; br. = branch; Pre-infund. = preinfundibular. Copyright Juan C. Fernandez-Miranda. Published with permission. Figure is available in color online only.

and perforating branches from the anterior communicating complex, with anastomoses between them, ${ }^{6,22,26}$ thus lowering the risk of chiasmal deficit after unilateral pSHA occlusion. However, as discussed above, we observed a significant distribution of the pSHA onto cisternal optic nerves, anterior chiasm, and even the anterior portion of optic tracts with minimal collateral or overlapping perfusion, thus confirming the findings of previous authors. ${ }^{4,6}$ Injury to the optic branches from the pSHA is more likely to cause a visual deficit, typically a superior quadrant defect when affecting the optic nerve or a homonymous hemianopia when affecting the optic tract.

There are limited reports concerning the visual outcome of SHA occlusion. Goto et al. reported a case in which a repeated loss of intraoperative flash visual evoked potentials followed occlusion of the SHA, and the occlusion was finally avoided and no visual deficit occurred. ${ }^{12}$ El Refaee et al. described two cases in which the SHAs were occluded during aneurysm clipping and intraoperative indocyanine green (ICG) angiography was used to ensure collateral filling of the occluded vessels. However, one case experienced superior quadrantanopia despite the retrograde filling. A homonymous hemianopia was reported after the SHA was occluded during aneurysm em- bolization with Onyx HD-500. ${ }^{15}$ Horiuchi et al. conducted a large series on visual outcome after clipping an SHA aneurysm: ${ }^{14}$ the overall incidence of a visual deficit was $13 \%$, among which 1 of 18 cases experienced visual loss despite SHA preservation, 2 of 22 cases had visual loss after sacrifice of the SHA, and 6 of 20 cases endured visual deficit with an undetermined condition of the SHA. In their series, however, the authors excluded carotid cave aneurysms, an area in which two-thirds of pSHAs originate, and data did not specify whether the SHAs were primary or secondary. This may potentially underestimate the true risk of SHA occlusion in SHA aneurysms.

While no definitive conclusion can be made regarding the visual outcome after pSHA occlusion, the overall impression is that it may be safe in a high percentage of cases due to overlapping blood supply and vascular anastomoses of the optic apparatus. The predictive value of intraoperative visual evoked potentials and ICG is still uncertain. Bilateral occlusion will carry a much higher risk of visual disturbance.

We suggest a hierarchy in sacrificing pSHA branches when necessary, based on the branching pattern and anastomoses of the SHAs. The descending branch should be sacrificed first as it anchors the SHA inferiorly and limits 

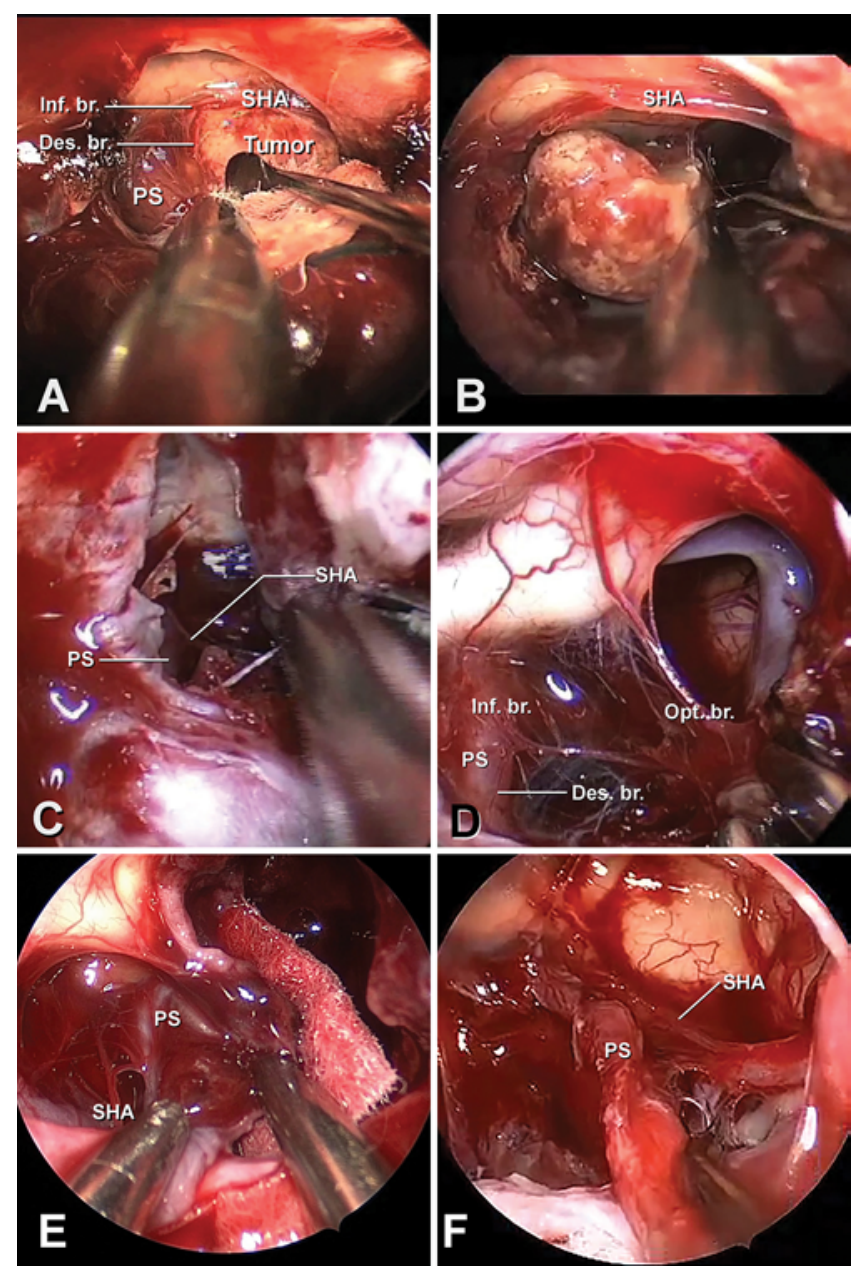

FIG. 6. Intraoperative visualization of SHA. Case 1. Descending branch tightly adhered to tumor (A). After sacrificing the descending branch, the optic chiasm could be dissected up, and more space was created to dissect the tumor (B). Case 2. After initial debulking, the SHA could be seen to be displaced posteriorly by the tumor (C). Well-preserved SHA and branches (D). Case 3. Well-preserved SHA on the right (E) and left (F). br. = branch; Des. = descending; Inf. = infundibular; Opt. = optic; PS $=$ pituitary stalk. Figure is available in color online only.

upward mobilization of the main stem of the SHA and the infundibular and optic branches; the descending branch is often just a meningeal branch, and when supplying the gland, its distribution is shared and anastomosed with the contralateral descending branch, the sinusoid vessels from circuminfundibular anastomosis, and the inferior hypophyseal arteries. ${ }^{23,25,29}$ The next branch to be considered is the infundibular branch, especially when the contralateral pSHA is not visibly hypoplastic since the preinfundibular anastomosis will compensate the loss of supply. This option carries a higher risk for endocrine deficit. The next option for sacrifice would be the main stem of a nondominant SHA near its origin from the ICA before branching since the contralateral pSHA may compensate via preinfundibular anastomosis. This option has a higher risk for both endocrine and visual deficit. Transection of the optic branch alone, however, may not be well compensated as there is a paucity of anastomosis with the superior chiasmal artery at this segment.

\section{Conclusions}

There are often two SHAs arising from each ICA. The pSHA is the proximal one, which frequently arises from the clinoidal ICA, and almost always supplies the infundibulum, optic chiasm, and proximal optic nerve. Unilateral injury to the pSHA is less likely to cause an endocrine deficit given the artery's universal preinfundibular anastomoses, but damage to the optic nerve branches can cause a visual deficit given the paucity of anastomosis between optic nerve branches. Selective sacrifice of the descending branches facilitates superior mobilization of the main stem of the SHA and its branches and may be well tolerated. A detailed understanding of the surgical anatomy of the SHA and its many variations may help surgeons when approaching challenging lesions in the suprasellar region.

\section{References}

1. Adams JH, Daniel PM, Prichard MML: Transection of the pituitary stalk in man: anatomical changes in the pituitary glands of 21 patients. J Neurol Neurosurg Psychiatry 29:545-555, 1966

2. Bergland R, Ray BS: The arterial supply of the human optic chiasm. J Neurosurg 31:327-334, 1969

3. Bergland RM, Page RB: Can the pituitary secrete directly to the brain? (Affirmative anatomical evidence). Endocrinology 102:1325-1338, 1978

4. Blunt MJ, Steele EJ: The blood supply of the optic nerve and chiasma in man. J Anat 90:486-493, 1956

5. Cavallo LM, de Divitiis O, Aydin S, Messina A, Esposito F, Iaconetta G, et al: Extended endoscopic endonasal transsphenoidal approach to the suprasellar area: anatomic considerations-part 1. Neurosurgery 62 (6 Suppl 3):1202-1212, 2008

6. Dawson BH: The blood vessels of the human optic chiasma and their relation to those of the hypophysis and hypothalamus. Brain 81:207-217, 1958

7. El Refaee EA, Baldauf J, Balau V, Rosenstengel C, Schroeder $\mathrm{H}$ : Is it safe to sacrifice the superior hypophyseal artery in aneurysm clipping? A report of two cases. J Neurol Surg A Cent Eur Neurosurg 74 (Suppl 1):e255-e260, 2013

8. Fernandez-Miranda JC, Gardner PA, Rastelli MM Jr, PerisCelda M, Koutourousiou M, Peace D, et al: Endoscopic endonasal transcavernous posterior clinoidectomy with interdural pituitary transposition. J Neurosurg 121:91-99, 2014

9. Gibo H, Kobayashi S, Kyoshima K, Hokama M: Microsurgical anatomy of the arteries of the pituitary stalk and gland as viewed from above. Acta Neurochir (Wien) 90:60-66, 1988

10. Gibo H, Koyama T, Koyama J, Ito K, Hokama M, Osawa M, et al: The superior hypophyseal artery: Microsurgical anatomy. Clin Neurol Neurosurg 99:S48, 1997

11. Gorczyca W, Hardy J: Arterial supply of the human anterior pituitary gland. Neurosurgery 20:369-378, 1987

12. Goto T, Tanaka Y, Kodama K, Kusano Y, Sakai K, Hongo K: Loss of visual evoked potential following temporary occlusion of the superior hypophyseal artery during aneurysm clip placement surgery. Case report. J Neurosurg 107:865-867, 2007

13. Hitotsumatsu T, Natori Y, Matsushima T, Fukui M, Tateishi J: Micro-anatomical study of the carotid cave. Acta Neurochir (Wien) 139:869-874, 1997

14. Horiuchi T, Goto T, Tanaka Y, Kodama K, Tsutsumi K, Ito K, et al: Role of superior hypophyseal artery in visual function impairment after paraclinoid carotid artery aneurysm surgery. J Neurosurg 123:460-466, 2015

15. Johnson JN, Elhammady M, Post J, Pasol J, Ebersole K, Aziz-Sultan MA: Optic pathway infarct after Onyx HD 500 
aneurysm embolization: visual pathway ischemia from superior hypophyseal artery occlusion. J Neurointerv Surg 6:e47, 2014

16. Joo W, Funaki T, Yoshioka F, Rhoton AL Jr: Microsurgical anatomy of the carotid cave. Neurosurgery 70 (2 Suppl Operative):300-312, 2012

17. Kim JM, Romano A, Sanan A, van Loveren HR, Keller JT: Microsurgical anatomic features and nomenclature of the paraclinoid region. Neurosurgery 46:670-682, 2000

18. Kobayashi S, Kyoshima K, Gibo H, Hegde SA, Takemae T, Sugita K: Carotid cave aneurysms of the internal carotid artery. J Neurosurg 70:216-221, 1989

19. Krisht AF, Barrow DL, Barnett DW, Bonner GD, Shengalaia G: The microsurgical anatomy of the superior hypophyseal artery. Neurosurgery 35:899-903, 1994

20. Leclercq TA, Grisoli F: Arterial blood supply of the normal human pituitary gland. An anatomical study normal human. J Neurosurg 58:678-681, 1983

21. Lee KF, Parke W, Lin SR, Choi HY, Schatz NJ: The vasculature of the diaphragma sellae. A postmortem injection study. Neuroradiology 16:281-283, 1978

22. Marinković SV, Milisavljević MM, Marinković ZD: Microanatomy and possible clinical significance of anastomoses among hypothalamic arteries. Stroke 20:1341-1352, 1989

23. McConnell EM: The arterial blood supply of the human hypophysis cerebri. Anat Rec 115:175-203, 1953

24. Moussazadeh N, Prabhu V, Bander ED, Cusic RC, Tsiouris AJ, Anand VK, et al: Endoscopic endonasal versus open transcranial resection of craniopharyngiomas: a casematched single-institution analysis. Neurosurg Focus 41(6):E7, 2016

25. Stanfield JP: The blood supply of the human pituitary gland. J Anat 94:257-273, 1960

26. van Overbeeke J, Sekhar L: Microanatomy of the blood supply to the optic nerve. Orbit 22:81-88, 2003

27. Wannemuehler TJ, Rubel KE, Hendricks BK, Ting JY, Payner TD, Shah MV, et al: Outcomes in transcranial micro- surgery versus extended endoscopic endonasal approach for primary resection of adult craniopharyngiomas. Neurosurg Focus 41(6):E6, 2016

28. Wislocki GB, King LS: The permeability of the hypophysis and hypothalamus to vital dyes, with a study of the hypophyseal vascular supply. Am J Anat 58:421-472, 1936

29. Xuereb GP, Prichard MM, Daniel PM: The arterial supply and venous drainage of the human hypophysis cerebri. Q J Exp Physiol Cogn Med Sci 39:199-217, 1954

\section{Disclosures}

The authors report no conflict of interest concerning the materials or methods used in this study or the findings specified in this paper.

\section{Author Contributions}

Conception and design: Fernandez-Miranda, Truong, ZanabriaOrtiz. Acquisition of data: Truong, Najera, Zanabria-Ortiz, Celtikci, Sun, Borghei-Razavi. Analysis and interpretation of data: Truong. Drafting the article: Truong. Critically revising the article: Fernandez-Miranda, Truong, Najera, Gardner. Reviewed submitted version of manuscript: all authors. Approved the final version of the manuscript on behalf of all authors: FernandezMiranda. Study supervision: Fernandez-Miranda.

\section{Supplemental Information \\ Videos \\ Video 1. https://vimeo.com/260947495. \\ Video 2. https://vimeo.com/260947606.}

\section{Correspondence}

Juan C. Fernandez-Miranda: University of Pittsburgh Medical Center, Pittsburgh, PA. fernandezmirandajc@upmc.edu. 